\title{
Low emittance muon accelerator studies with production from positrons on target
}

\author{
M. Boscolo, ${ }^{1, *}$ M. Antonelli, ${ }^{1}$ O. R. Blanco-García, ${ }^{1}$ S. Guiducci, ${ }^{1}$ S. Liuzzo, ${ }^{2}$ \\ P. Raimondi, ${ }^{2}$ and F. Collamati ${ }^{3}$ \\ ${ }^{1}$ INFN-LNF, Via E. Fermi 40, 00044 Frascati, Rome, Italy \\ ${ }^{2}$ ESRF, 71 avenue des Martyrs, 38000 Grenoble, France \\ ${ }^{3}$ INFN-Rome, Piazzale A. Moro 2, 00185 Rome, Italy
}

(Received 14 March 2018; published 26 June 2018)

\begin{abstract}
A new scheme to produce very low emittance muon beams using a positron beam of about $45 \mathrm{GeV}$ interacting on electrons on target is presented. One of the innovative topics to be investigated is the behaviour of the positron beam stored in a low emittance ring with a thin target, that is directly inserted in the ring chamber to produce muons. Muons can be immediately collected at the exit of the target and transported to two $\mu^{+}$and $\mu^{-}$accumulator rings and then accelerated and injected in muon collider rings. We focus in this paper on the simulation of the $\mathrm{e}^{+}$beam interacting with the target, the effect of the target on the 6-D phase space and the optimization of the $\mathrm{e}^{+}$ring design to maximize the energy acceptance. We will investigate the performance of this scheme, ring plus target system, comparing different multi-turn simulations. The source is considered for use in a multi-TeV collider in [F. Zimmermann, in Strategy for Future Extreme Beam Facilities, Accelerator Technology Institute of Electronic Systems, Vol. 44 (Warsaw University of Technology, 2017).].
\end{abstract}

DOI: 10.1103/PhysRevAccelBeams.21.061005

\section{INTRODUCTION}

Muon beams are customarily obtained via $K / \pi$ decays produced in proton interaction on target. A complete design study using this scheme, including the muon cooling system has been performed by the Muon Accelerator Program [1,2]. In this paper we will investigate the possibility to produce low emittance muon beams from a novel approach, using electron-positron collisions at a center-of-mass energy just above the $\mu^{+} \mu^{-}$production threshold with minimal muon energy spread, corresponding to the direct annihilation of approximately $45 \mathrm{GeV}$ positrons and atomic electrons in a thin target, $\mathrm{O}(0.01 \sim$ radiation lengths $)$. Concept studies on this subject are reported in Refs. [3,4]. A feasibility study of a muon collider based on muon electro-production has been studied in Ref. [5]. The most important key properties of the muons produced by the positrons on target are (i) the low and tunable muon momentum in the centre of mass frame; (ii) large boost, being about $\gamma \sim 200$. These characteristics result in the following advantages: (i) the final state muons are highly collimated and have very small emittance, overcoming the need to cool them; (ii) the muons have an average

\footnotetext{
"manuela.boscolo@lnf.infn.it
}

Published by the American Physical Society under the terms of the Creative Commons Attribution 4.0 International license. Further distribution of this work must maintain attribution to the author(s) and the published article's title, journal citation, and DOI. laboratory lifetime of about $500 \mu \mathrm{s}$ at production. (iii) the muons are produced with an average energy of $22 \mathrm{GeV}$ easing the acceleration scheme.

The use of a low emittance positron beam on target allows the production of low emittance muon beam and, unlike previous designs, muon cooling would not be necessary.

The very small emittance could enable high luminosity with smaller muon fluxes, reducing both the machine background in the experiments and more importantly the activation risks due to neutrino interactions.

Figure 1 shows the dose equivalent due to neutrino radiation as taken from [6]. It shows an increase of the dose equivalent with the muon beam energy posing a severe limit on the center of mass energy reach of the muon collider. It has been obtained for a muon rate of $3 \times 10^{13} \mathrm{~s}^{-1}$ and a collider depth of $100 \mathrm{~m}$. Such a muon rate is problematic for muon collider operations above about $6 \mathrm{TeV}$ center of mass energy [1]. Reference [1] chose a collider depth of $500 \mathrm{~m}$ for a $6 \mathrm{TeV}$ collider scenario.

Figure 1 and Ref. [6] provides a first crude approximation of the dose effect, and a more detailed calculation with consideration of mitigation strategies is needed for precise limit. To enable higher centre of mass energies the muon rate has to be reduced, thus competitive luminosity performance must be obtained by reducing the beam emittance.

The current muon collider design studied by MAP [1] foresees a normalized emittance of $25 \mu \mathrm{m}$ with a luminosity of about $10^{35} \mathrm{~cm}^{-2} \mathrm{~s}^{-1}$ at $6 \mathrm{TeV}$. The muons per bunch are $2 \times 10^{12}$ and the muon production rate is $3 \times 10^{13} \mathrm{~s}^{-1}$. 


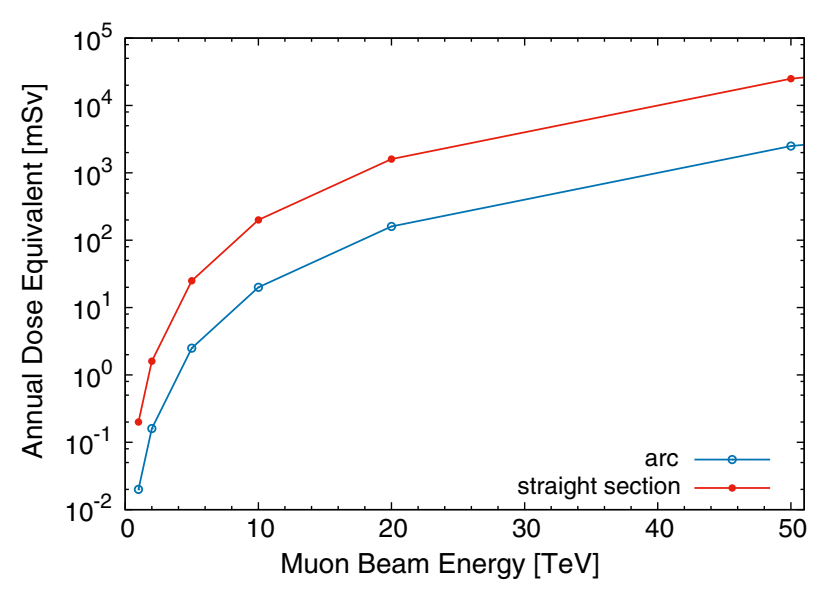

FIG. 1. Dose equivalent due to neutrino radiation at a collider depth of $100 \mathrm{~m}$ for a muon rate of $3 \times 10^{13} \mathrm{~s}^{-1}$ as taken from [6]. Contributions from the straight section and from the arc are shown in red and blue, respectively.

The aim of the study is to obtain comparable luminosity performances with lower fluxes and smaller emittances allowing operation at a higher center of mass energy. One possibility is to accommodate the accelerator complex in the CERN area in existing tunnels. A preliminary proposal for a $14 \mathrm{TeV}$ CERN muon collider has been described in Ref [1].

\section{A. Muon production}

The cross section for continuum muon pair production $e^{+} e^{-} \rightarrow \mu^{+} \mu^{-}$has a maximum value of about $1 \mu \mathrm{b}$ at $\sqrt{s} \sim 0.230 \mathrm{GeV}$. In our proposal these values of $\sqrt{s}$ can be obtained from fixed target interactions with a positron beam energy of

$$
E\left(e^{+}\right) \approx \frac{s}{2 m_{e}} \approx 45 \mathrm{GeV}
$$

where $m_{e}$ is the electron mass, with a boost of

$$
\gamma \approx \frac{E\left(e^{+}\right)}{\sqrt{s}} \approx \frac{\sqrt{s}}{2 m_{e}} \approx 220 .
$$

The maximum scattering angle of the outgoing muons $\theta_{\mu}^{\max }$ depends on $\sqrt{s}$. In the approximation of $\beta_{\mu}=1$,

$$
\theta_{\mu}^{\max }=\frac{4 m_{e}}{s} \sqrt{\frac{s}{4}-m_{\mu}^{2}}
$$

where $\beta_{\mu}$ is the muon velocity. Muons produced with very small momentum in the rest frame are well contained in a cone of about $5 \times 10^{-4} \mathrm{rad}$ for $\sqrt{s}=0.212 \mathrm{GeV}$, the cone size increases to $\sim 1.2 \times 10^{-3} \mathrm{rad}$ at $\sqrt{s}=0.220 \mathrm{GeV}$. The difference between the maximum and the minimum energy of the muons produced at the positron target $\left(\Delta E_{\mu}\right)$ also depends on $\sqrt{s}$, and with the $\beta_{\mu}=1$ approximation we get:

$$
\Delta E_{\mu}=\frac{\sqrt{s}}{2 m_{e}} \sqrt{\frac{s}{4}-m_{\mu}^{2}}
$$

The RMS energy distribution of the muons increases with $\sqrt{s}$, from about $1 \mathrm{GeV}$ at $\sqrt{s}=0.212 \mathrm{GeV}$ to $3 \mathrm{GeV}$ at $\sqrt{s}=0.220 \mathrm{GeV}$.

\section{B. Target options}

The number of $\mu^{+} \mu^{-}$pairs produced per positron bunch on target is

$$
n\left(\mu^{+} \mu^{-}\right)=n^{+} \rho^{-} L \sigma\left(\mu^{+} \mu^{-}\right)
$$

where $n^{+}$is the number of positrons in the bunch, $\rho^{-}$is the electron density in the medium, $L$ is the thickness of the target, and $\sigma\left(\mu^{+} \mu^{-}\right)$is the muon pairs production cross section. The dominant process in $e^{+} e^{-}$interactions at these energies is collinear radiative Bhabha scattering, with a cross section of about $\sigma_{r b} \approx 150 \mathrm{mb}$ for a positron energy loss larger than $1 \%$. This sets the value of the positron beam interaction length for a given pure electron target density value. So, it is convenient to use targets with thickness of at most one interaction length, corresponding to $L=1 /\left(\sigma_{r b} \rho^{-}\right)$:

$$
\left(\rho^{-} L\right)_{\max }=1 / \sigma_{r b} \approx 10^{25} \mathrm{~cm}^{-2}
$$

The ratio of the muon pair production cross section to the radiative bhabha cross section determines the maximum value of the muon conversion efficiency $\operatorname{eff}\left(\mu^{+} \mu^{-}\right)$that can be obtained with a pure electrons target. In the following we will refer to eff $\left(\mu^{+} \mu^{-}\right)$defined as the ratio of the number of produced $\mu^{+} \mu^{-}$pairs to the number of the incoming positrons. One can easily see that the upper limit of $\operatorname{eff}\left(\mu^{+} \mu^{-}\right)$is of the order of $10^{-5}$, so that:

$$
n\left(\mu^{+} \mu^{-}\right)_{\max } \approx n^{+} 10^{-5} \text {. }
$$

Electromagnetic interactions with nuclei are dominant in conventional targets. In addition, limits are present for the target thickness not to increase the muon beam emittance $\epsilon_{\mu}$. Assuming a uniform distribution in the transverse $x-x^{\prime}$ plane the emittance contribution due to the target thickness is proportional to the target length and to the maximum scattering angle of the outgoing muons:

$$
\epsilon_{\mu} \propto L\left(\theta_{\mu}^{\max }\right)^{2}
$$

The number of $\mu^{+} \mu^{-}$pairs produced per crossing has the form given by Eq. (5), with

$$
\rho^{-}=N_{A} / A \rho Z
$$

where $Z$ is the atomic number, $A$ the mass number, $N_{A}$ Avogadro's constant, and $\rho$ the material density. In addition, the multiple scattering contributes to the emittance increase according to: 


$$
\theta_{\text {r.m.s }} \sim \frac{13.6 \mathrm{MeV}}{\beta c p} \sqrt{x / X_{0}}\left[1+0.038 \ln \left(x / X_{0}\right)\right]
$$

where $p$ and $\beta c$ are the positron velocity and momentum, respectively, $x / X_{0}$ is the target thickness in radiation lengths. Similarly:

$$
x_{\text {r.m.s }} \sim \theta_{\text {r.m.s }} 0.5 L \sqrt{3} .
$$

The bremsstrahlung process governs the positron beam degradation in this case and it scales with the radiation length. Roughly, the cross section per atom increases by a factor $(Z+1)$ with respect to the case of a pure electron target.

On one side to minimize the emittance there is the need of a small length $L$ (thin target), on the other side compact materials have typically small radiation lengths causing an increase of the emittance due to multiple scattering and a fast positron beam degradation due to bremsstrahlung. Positron interactions on different targets have been studied with GEANT4 [7]. The results show that the optimal target has to be thin and not too heavy [3]. Carbon (C), Beryllium (Be) and Liquid Lithium (LLi) have the best performances.

A $3 \mathrm{~mm}$ Beryllium target provides a muon conversion efficiency eff $\left(\mu^{+} \mu^{-}\right)$of about $0.7 \times 10^{-7}$. The muon transverse phase space at the target exit, as produced by a $45 \mathrm{GeV}$ positron beam, is shown in Fig. 2, assuming negligible emittance of the positron beam. A transverse emittance as small as $0.19 \times 10^{-9} \mathrm{~m}$-rad is observed for the outgoing $\sim 22 \mathrm{GeV}$ muons; it represents the ultimate value of emittance that can be obtained with such a scheme.

The very low muon production efficiency, due to the low value of the production cross section, makes convenient a scheme where positrons are recirculated after the interaction

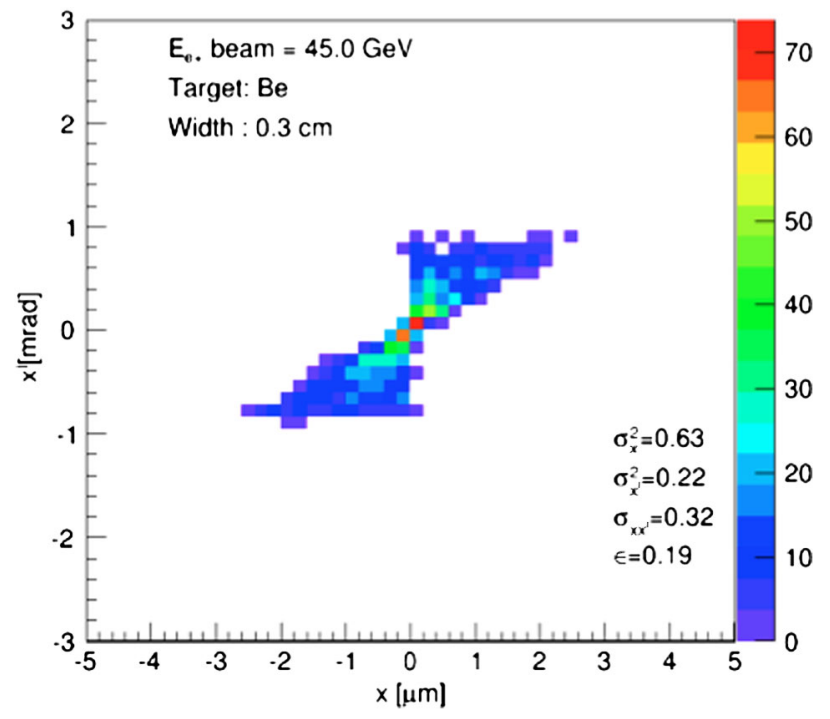

FIG. 2. Transverse phase space distribution of muons at the target exit (GEANT4). The positron beam impinging on the $3 \mathrm{~mm}$ Be target has negligible emittance. on a thin target. A $3 \mathrm{~mm}$ Beryllium target is considered in our studies.

A preliminary layout of Low EMittance Muon Accelerator (LEMMA) is shown in Fig. 3. This scheme foresees muons produced by the interaction of positrons circulating in a storage ring on target at $\sim 22 \mathrm{GeV}$, then muons are accumulated in isochronous rings with a circumference of $\sim 60 \mathrm{~m}$ with $13 \mathrm{~T}$ dipoles. In addition to muons, high intensity and high energy photons are produced from the interaction of the positron beam with the target. The possibility of using these photons for an embedded positron source is extremely appealing and will be briefly described in Sec. IV. However, a solution to transform the temporal structure of produced positrons to be used for the ring injection has not been found yet.

This innovative scheme has many key topics to be investigated: (i) a low emittance high energy acceptance $45 \mathrm{GeV}$ positron ring, (ii) $\mathrm{O}(100 \mathrm{~kW})$ class target, (iii) high momentum acceptance muon accumulator rings, (iv) high rate positron source.

A first design of a $45 \mathrm{GeV}$ positron ring with low emittance and high momentum acceptance will be described in the following. The effects on beam parameters due to the target insertion will be analysed from the point of view of the positron beam lifetime and its degradation. In particular, an attempt to the control the positron emittance growth will be described. Our goal is to preserve as much as possible the ultimate value of normalized emittance, $\epsilon_{\mu}=40 \mathrm{~nm}$, obtained for a $3 \mathrm{~mm}$ Be target and to obtain a positron beam lifetime of about 250 turns. Additional emittance dilution effects due to the muon accumulation are the subject of future studies.

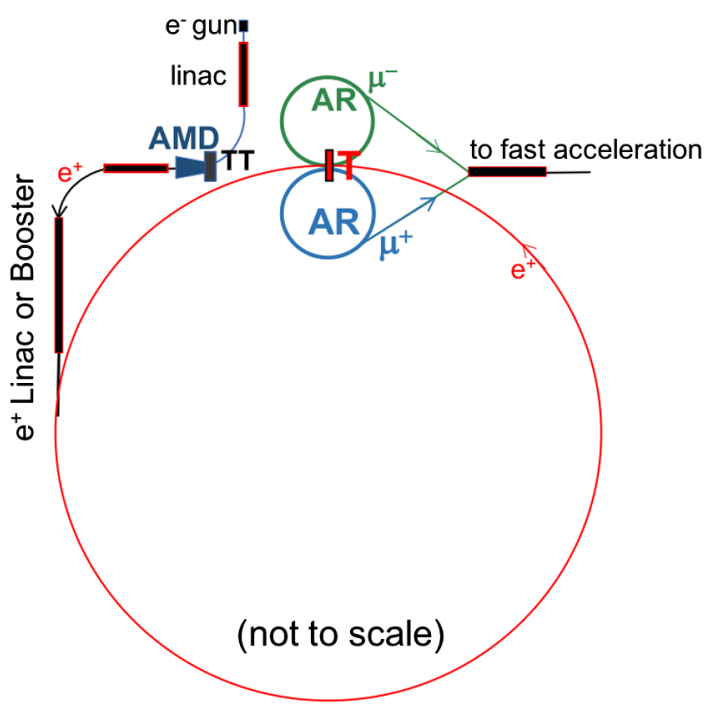

FIG. 3. Schematic layout for low emittance muon beam production: positron source with adiabatic matching device (AMD), $e^{+}$ring plus target (T) for $\mu^{+}-\mu^{-}$production, two $\mu^{+}-$ $\mu^{-}$accumulator rings (AR), and fast acceleration section to be followed by the muon collider. 
TABLE I. Positron ring parameters.

\begin{tabular}{lcc}
\hline \hline Parameter & Units & \\
\hline Energy & $\mathrm{GeV}$ & 45 \\
Circumference (32 ARCs, no IR) & $\mathrm{m}$ & 6300.960 \\
Geometrical emittance x, y & $\mathrm{m}$ & $5.73 \times 10^{-9}$ \\
Bunch length & $\mathrm{mm}$ & 3 \\
Beam current & $\mathrm{mA}$ & 240 \\
rf frequency & $\mathrm{MHz}$ & 500 \\
rf voltage & $\mathrm{GV}$ & 1.15 \\
Harmonic number & $\#$ & 10508 \\
Number of bunches & $\#$ & 100 \\
No. of particles/bunch & $\#$ & $3.15 \times 10^{11}$ \\
Synchrotron tune & & 0.068 \\
Transverse damping time & $\mathrm{turns}$ & 175 \\
Longitudinal damping time & turns & 87.5 \\
Energy loss/turn & $\mathrm{GeV}$ & 0.511 \\
Momentum compaction & & $1.1 \times 10^{-4}$ \\
rf acceptance & $\%$ & \pm 7.2 \\
Energy spread & $\mathrm{dE} / \mathrm{E}$ & $1 \times 10^{-3}$ \\
SR power & $\mathrm{MW}$ & 120 \\
\hline \hline
\end{tabular}

\section{POSITRON STORAGE RING}

A $45 \mathrm{GeV}$ low emittance positron storage ring has been designed and the effect of the target on the beam properties has been studied. The main processes affecting the beam sizes in the target are bremsstrahlung and multiple Coulomb scattering. The effects of these two contributions have been studied separately, finding that the best location for the target corresponds to a low- $\beta$, dispersion-free region.

The ring is composed of 32 lattice cells of $197 \mathrm{~m}$ each for a total length of $6 \mathrm{~km}$ with the parameters shown in Table I. The cell shown in Fig. 4 is based on the hybrid multibend achromat (HMBA) [8] to minimize emittance, while keeping large momentum and dynamic acceptance. The maximum dipole field is $0.26 \mathrm{~T}$, the filling factor is $77 \%$.

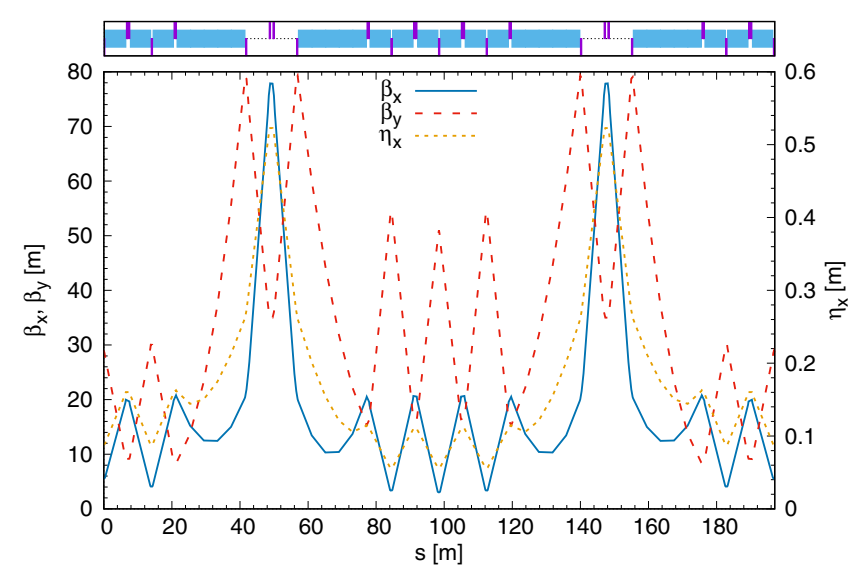

FIG. 4. Optical functions of one cell of the $45 \mathrm{GeV} \mathrm{e}^{+}$ring. $\beta_{x}$ and $\beta_{y}$ are shown in blue and dotted red, respectively; horizontal dispersion function $\eta_{x}$ is plotted in yellow with values on the right y axis.
The maximum quadrupole, sextupole, and octupole gradients are $110 \mathrm{~T} / \mathrm{m}, 340 \mathrm{~T} / \mathrm{m}^{2}$, and $5900 \mathrm{~T} / \mathrm{m}^{3}$ respectively. The horizontal and vertical phase advance, in $2 \pi$ units, between sextupoles at the peak of the horizontal dispersion is 1.5 and 0.5 respectively. Sixty-four rf cavities have been considered, each rf cavity is $5.4 \mathrm{~m}$ long and composed by 9 rf cells of about $7 \mathrm{MV} / \mathrm{m}$ accelerating gradient. Transverse unnormalized emittance is $5.73 \times 10^{-9}$ and longitudinal emittance $3 \mu \mathrm{m}$; then, as the positron beam passes through the target, emittance is increased as discussed later, see Fig. 11. Longitudinal emittance is increased due to a large elongation effect due to the bremsstrahlung effect in the passage of the target.

The effect of the target on the positron beam has been studied for different locations in the cell for different materials and thicknesses. This simulation has been divided in two parts: particle tracking and positron interaction with target. Particle tracking in the ring is performed with Accelerator Toolbox (AT) [9] and MAD-X PTC [10], while positron interaction with the target is studied with either GEANT4 or FLUKA [11].

The cycle starts by the generation of a particle distribution from the equilibrium emittances of the ring. These particles are tracked through the ring for one turn and the particle distribution is modified according to simulations to account for the passage through the target. Radiation damping is included in the simulation. Single turn tracking and target interaction are then repeated for a given number of turns.

Coulomb scattering in the target changes the beam divergence and the beam size. The multiple Coulomb scattering contribution is normally distributed and uncorrelated with the beam, therefore it only depends on the target. It can be estimated as in Eq. (9). As the beam passes through the target several times, the contribution from multiple scattering (MS) increases as

$$
\sigma_{x^{\prime}, y^{\prime}}(M S)=\sqrt{N} \theta_{\text {r.m.s }}
$$

where $N$ is the turn number. Therefore, the beam divergence per turn is given by

$$
\sigma_{x^{\prime}, y^{\prime}}(N)=\sqrt{\sigma_{x^{\prime}, y^{\prime}}^{2}(M S)+\sigma_{x^{\prime}, y^{\prime}}^{2}(0)}
$$

where $\sigma_{x^{\prime}, y^{\prime}}(0)$ is the unperturbed beam divergence in the two transverse planes.

Similar effects have been studied for ionization cooling [12] and for ion stripping [13].

While the contribution to the divergence from multiple scattering is completely determined by the target, the contribution to the beam size is expected to be proportional to the $\beta$-function at the target location.

At a waist the beam width after $N$ machine turns is given by 

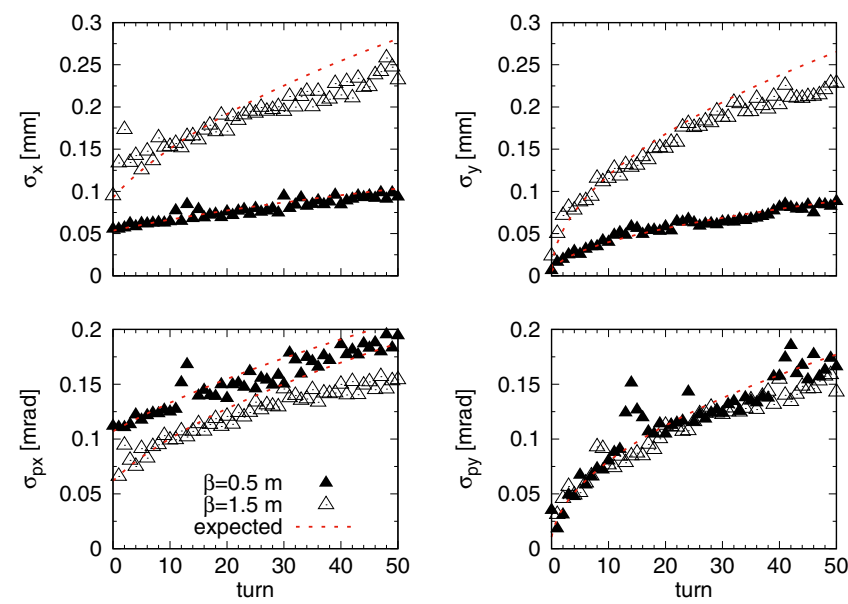

FIG. 5. Positron beam transverse evolution vs machine turns due to multiple scattering with a $3 \mathrm{~mm}$ Be target. Triangles are PTC tracking results, for two $\beta^{*}$ values at the IP $(0.5$ and $1.5 \mathrm{~m})$, while dashed lines are the expected behaviour from equations (10) and (11). An emittance ratio of $1 \%$ has been considered for this simulation.

$$
\sigma_{x, y}(N)=\beta_{x, y} \sigma_{x^{\prime}, y^{\prime}}(N) .
$$

This explains how a strong reduction of the beam size growth is obtained by placing the target in a low- $\beta$ location, such as the interaction point in a collider ring. To visualize this effect, Fig. 5 shows the beam size and divergence for a positron beam interacting with a $3 \mathrm{~mm}$ thick Beryllium target at each turn. In the case of $\beta$ value at the target (waist) equal to $1.5 \mathrm{~m}$ the simulated increase in the beam divergence is $\theta_{\text {r.m.s }} \sim 25 \mu \mathrm{rad}$ at each single pass. Lower $\beta$ functions evidently reduce this effect.

Minimization of the emittance growth is obtained by placing the target in a beam waist and setting the angular contribution from the multiple scattering similar to or smaller than the beam divergence [14]. This matching reduces beam filamentation [15].

Bremsstrahlung in the target causes the particles to lose energy, degrading the beam emittance and reducing its lifetime. For a $3 \mathrm{~mm}$ thick target of Beryllium we obtain, from tracking with MAD-X PTC, a lifetime between 37 and 40 turns, equivalent to $0.8 \mathrm{~ms}$, in good agreement with AT results. Figure 6 shows the number of particles per turn for several target thicknesses from which it is concluded that the beam lifetime and target thickness are inversely proportional, as expected.

Beam lifetime slightly increases due to radiation damping. For a $3 \mathrm{~mm}$ Be target the effect is negligible because the ring longitudinal damping time is approximately twice the 40 turns we have shown, and the transverse damping time is almost four times larger. With a Be target of $500 \mu \mathrm{m}$ the increase in beam lifetime amounts to $8 \%$ of the 140 turns obtained from simulations with MAD-X, is as shown in Fig. 7.

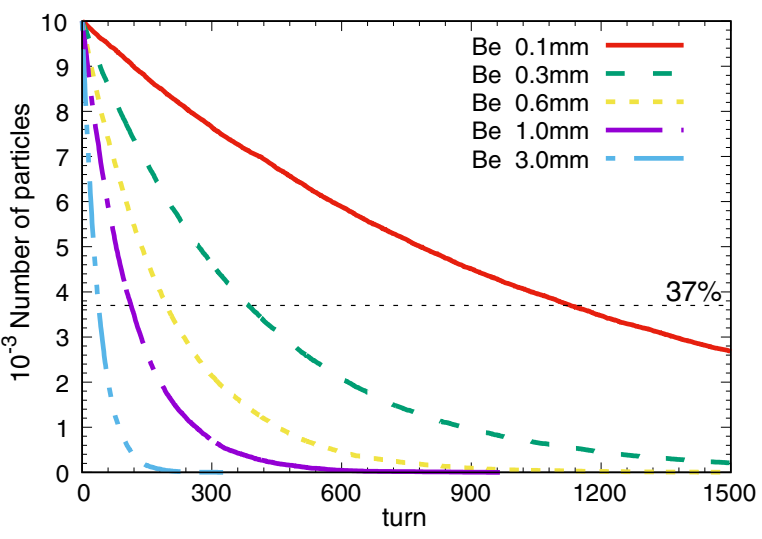

FIG. 6. Number of $\mathrm{e}^{+}$vs machine turns for various Be target thicknesses (MAD-X PTC). For a $3 \mathrm{~mm}$ Be target (light blue line) lifetime is about 40 turns.

Lifetime is limited by the energy loss, thus it is important to maximize the ring momentum acceptance. Figure 8 shows that the cell without errors reaches $8 \%$ of momentum acceptance, obtained from particle tracking along the ring with three different tracking codes: AT, MAD-X, and MAD-X PTC. Good agreement is found between the codes.

One of the ring cells has been modified to obtain a low- $\beta$ interaction region to place the target at its center similarly to the interaction point of a collider. Figure 9 shows the optics and layout of the current target insertion region and $25 \mathrm{~m}$ of

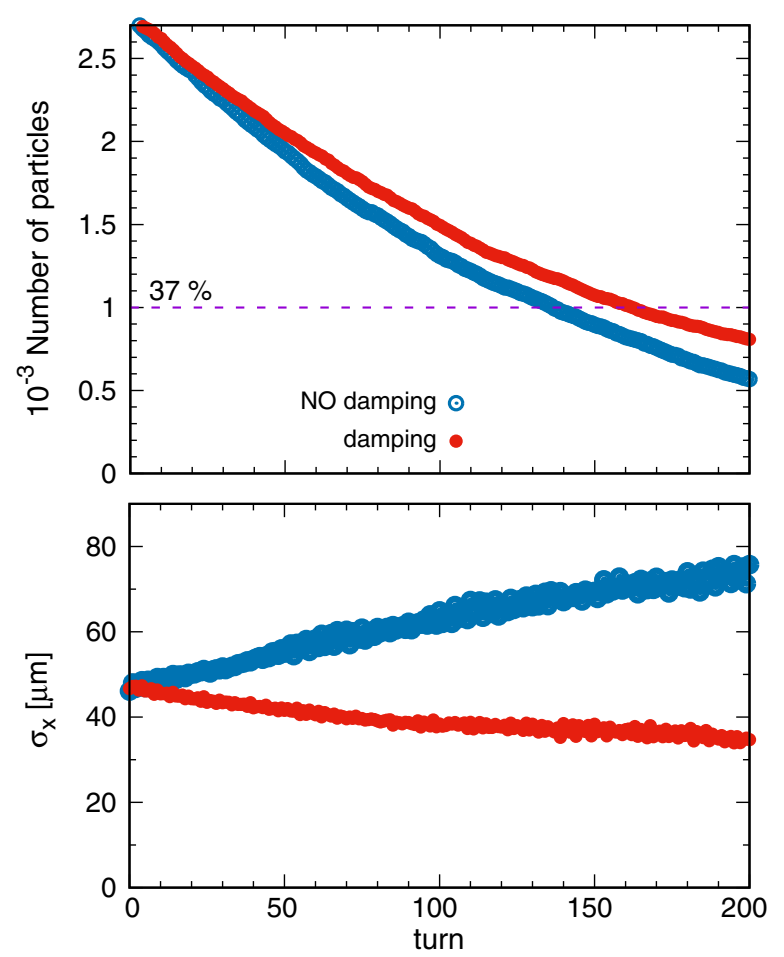

FIG. 7. Beam lifetime (top) and horizontal beam size (bottom) with and without the damping effect in the tracking simulation including a $0.5 \mathrm{~mm}$ thick Be target. 


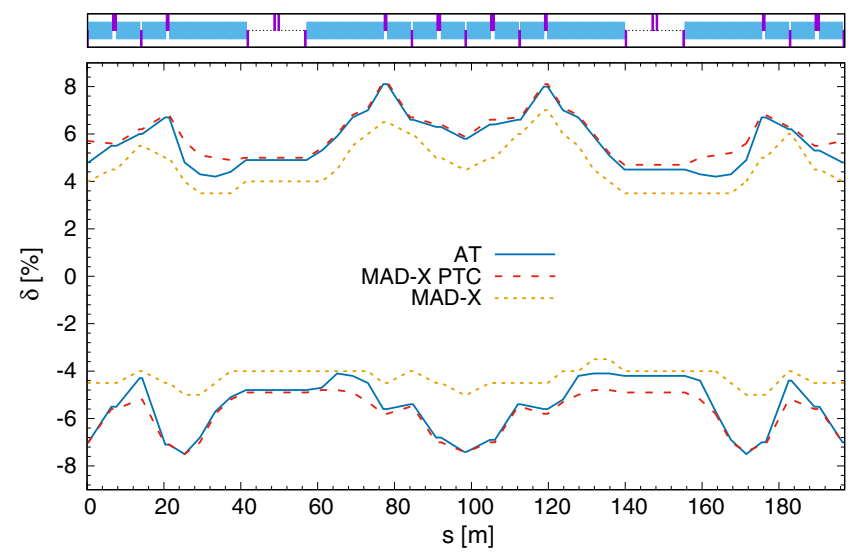

FIG. 8. Momentum acceptance of the positron ring cell obtained from tracking with MAD-X (dotted yellow), MAD-X PTC (dotted red), and AT (light blue). The best agreement is obtained between AT and MAD-X PTC.

the cell. At the target location, $s=0 \mathrm{~m}$, the optical functions are $\beta_{x}=\beta_{y}=0.5 \mathrm{~m}$ and $\eta_{x}=0 \mathrm{~mm}$.

In addition, linear and nonlinear terms related to the momentum deviation, $\delta p$, should be minimized because they contribute to the emittance growth. Figure 10 shows the positron beam size at the target increasing as a function of the machine turns for different values of dispersion function in the target insertion region, and with $\beta$ functions unchanged. When the dispersion at the target is cancelled, the beam size increase due to target interactions is damped.

Figure 11 shows the results of individual contributions to the beam emittance degradation from multiple scattering and bremsstrahlung. Values have been calculated using the 95\% core of the particle distribution. The current design status amounts only to a two-fold horizontal emittance increase and a four-fold in vertical by the end of the beam lifetime. The bremsstrahlung energy loss in the target gives a longitudinal emittance growth. This increase of emittance and corresponding bunch lengthening can be reduced by decreasing the ring momentum compaction and increasing

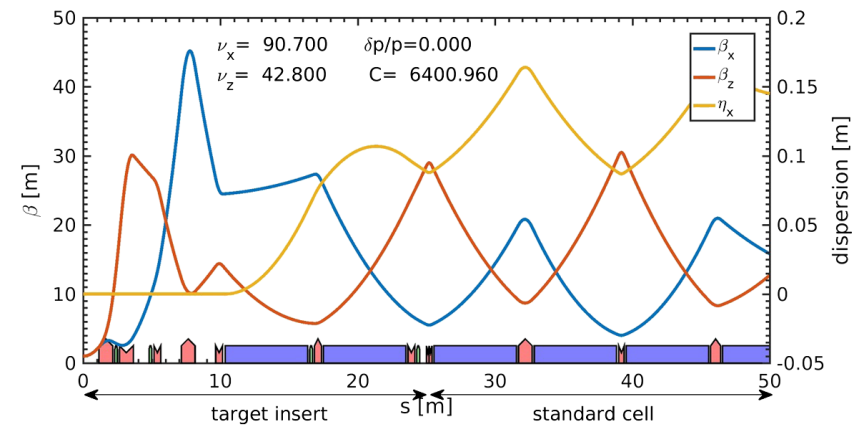

FIG. 9. Target insertion region (target is at $s=0 \mathrm{~m}$ ) and $25 \mathrm{~m}$ of the cell. $\beta_{x}$ and $\beta_{y}$ functions are shown in blue and red, respectively; horizontal dispersion function $\eta_{x}$ is plotted in yellow with values on the right $y$ axis.
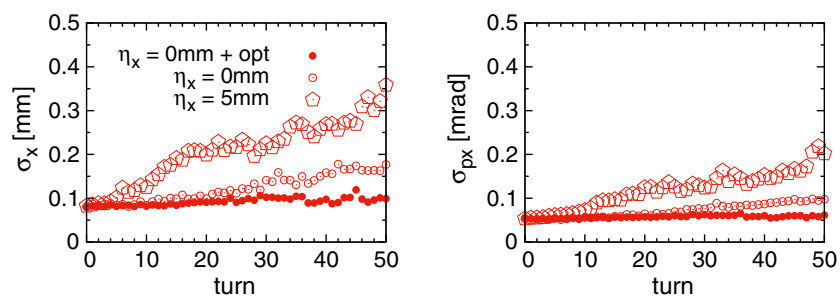

FIG. 10. Effect of the cancellation of the horizontal dispersion at the target location and optimization of the lattice on the beam size growth and divergence from bremsstrahlung. Full red dot markers show the best case with no degradation of $\sigma_{x}$ and $\sigma_{x^{\prime}}$, where linear and nonlinear dispersion terms at target are set to zero.

the rf voltage. The longitudinal emittance growth is completely dominated by the energy loss per turn, which makes the beam longer.

Thin low $\mathrm{Z}$ targets (i.e., thickness of the order of 0.01 $\mathrm{Xo}$ ) based on $\mathrm{Li}, \mathrm{Be}$ and $\mathrm{C}$ could be used for low emittance muon production. For equivalent electron density in the target, lighter materials will provide smaller beam perturbations at the cost of larger intrinsic muon beam emittances. Figure 12 shows the number of survived positrons as a function of machine turns for different material targets.

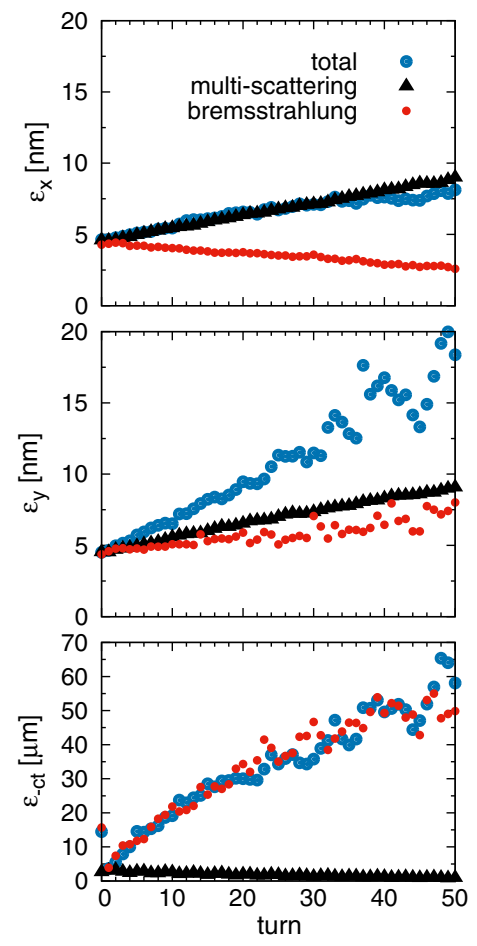

FIG. 11. Horizontal (upper plot), vertical (middle plot) and longitudinal (lower plot) emittance degradation as a function of turns is shown (blue dots) for a $3 \mathrm{~mm}$ thick Beryllium target with $\beta_{x}^{*}=\beta_{y}^{*}=0.5 \mathrm{~m}$. Contributions from multiple scattering (black triangles) and bremsstrahlung (red dots) are shown also separately. The horizontal emittance is dominated by multiplescattering; the longitudinal emittance by bremsstrahlung. 


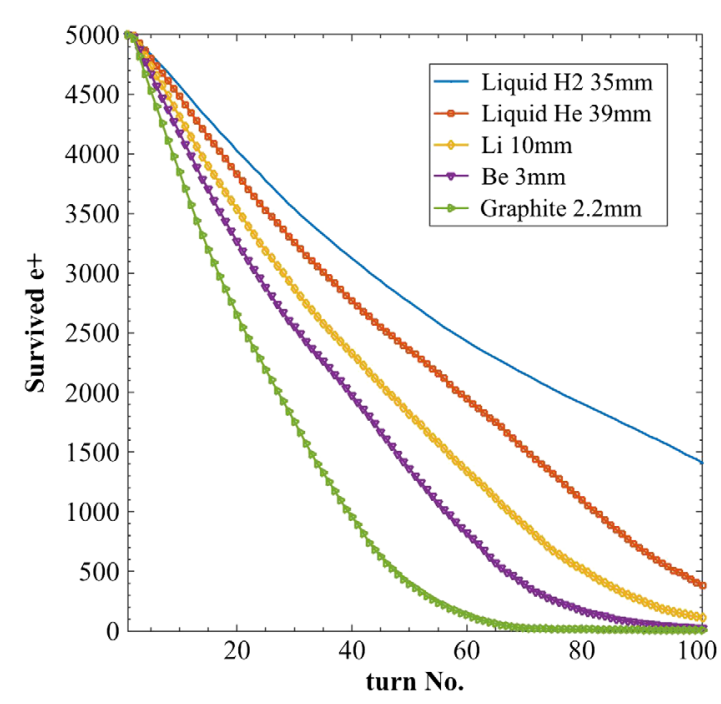

FIG. 12. Number of $\mathrm{e}^{+}$vs turns number for different target materials. Target thickness has been chosen to obtain a constant muon yield. $3 \mathrm{~mm} \mathrm{Be}$, shown in this plot in dark violet, is the case studied in our beam dynamics simulations.

A $10 \mathrm{~mm}$ Lithium target might provide sizeably larger lifetime at the cost of a factor three increase in the intrinsic muon beam emittance. To maximize the brilliance of the muon beam the positron beam spot at the target has to be minimized and the positron beam intensity maximized.

\section{TARGET CONSIDERATION}

Both temperature rise and thermal shock are related to the beam size on target. For a given material the lower limit on the beam size is obtained when there is no pile-up of bunches on the same target position. For this reason both the target and the positron beam have to be movable. A fast beam bump can be done after the extraction of one muon bunch from the muon accumulator ring every one muon laboratory lifetime, corresponding to approximately 2500 positron bunches. Fast moving targets can be obtained with rotating disks for solid targets or high velocity jets for liquids. A beam current of about $200 \mathrm{~mA}$ will provide about $100 \mathrm{~kW}$ of power that has to be removed to keep the target temperature under control. Be and C composites/structures are in use and under study for low $\mathrm{Z}$ target and collimators in accelerators for high energy physics also because of the stringent vacuum requirements in such complexes that are not easy to fulfil with $\mathrm{Li}$ targets. Recently developed $\mathrm{C}$ based materials with excellent thermo-mechanical properties are under study for the LHC upgrade collimators [16]. A $7.5 \mu \mathrm{s}$ long beam pulse made of 288 bunches with $1.2 \times 10^{11}$ protons per bunch, which is the full LHC injection batch extracted from SPS, has been used to test both C-based [16] and Be-based [17] targets with maximum temperatures reaching $1000^{\circ} \mathrm{C}$. Good results have been obtained with a beam spot of $0.3 \times 0.3 \mathrm{~mm}^{2}$. Liquid Lithium, LLi, is under study for divertors in tokamaks and in use for neutron production. In particular, LLi jets in the order of $1 \mathrm{~cm}$ thickness are used as targets for $\mathrm{MeV}$ proton beams to produce high flux neutron beams. Power deposited on target of about $0.5 \mathrm{MW}$ and of $>1 \mathrm{MW}$ have been obtained or planned with LLi jet velocities of $5 \mathrm{~m} / \mathrm{s}$ or $30 \mathrm{~m} / \mathrm{s}$ respectively [18]. In addition to evaporation, beam spot sizes smaller than $1 \mathrm{~mm}^{2}$ would reach the bubbling regime for LLi. One possibility to overcome this problem is to produce a fast LLi jet in a thin pipe.

\section{POSITRON SOURCE REQUIREMENTS}

The scheme described in the previous section relies on the possibility of enhancing the muon production by recirculating the positron beam, allowing multiple beam target interactions. However, every time the primary beam interacts in the target, positrons will lose part of their energy, and a certain fraction of them will exceed the energy acceptance of the machine, being eventually lost. An high intensity positron source is needed to replace these losses.

The present record positron production rate has been reached at the SLAC linac SLC. A summary of the parameters of the positron sources for the future facilities is reported in Table II. The ILC positron source has been designed to provide $3.9 \times 10^{14} \mathrm{e}^{+} / \mathrm{s}$. Two order of magnitudes more intense sources are foreseen for $\mathrm{LHeC}$. $\mathrm{LHeC}$ is an electron machine although a positron option has been conceived.

The required intensity is strongly related to the beam lifetime that is determined by the ring momentum acceptance and the target material, see Secs. II and III. The beam lifetime with the present optics (energy acceptance about $6 \%$ ) is in the range of 40-50 turns corresponding to about $2 \%-3 \%$ of positrons lost per turn. By increasing the ring circumference we aim at increasing the energy acceptance.

With 50 turns lifetime, the scenario requires $3 \times 10^{16}$ $45 \mathrm{GeV} e^{+} / s$. This corresponds to a beam power of 216 MW. A lifetime of 100 turns can be obtained with an $\mathrm{H}$ pellet target and energy acceptance of about $10 \%$.

In the interaction of the primary positron beam with the Beryllium target, bremsstrahlung photons are produced with a strong boost along the primary beam direction. It may then possible to exploit this photon flux for an

TABLE II. Positron sources parameters for future projects from Ref. [19]. The values given for LEMMA refer to the goal performances.

\begin{tabular}{|c|c|c|c|c|c|c|}
\hline & SLC & CLIC & ILC & LHeC I & $\mathrm{HeC} \mathrm{E}$ & MMA goal \\
\hline $\mathrm{E}[\mathrm{GeV}]$ & 1.19 & 2.86 & 4 & 140 & 60 & 45 \\
\hline$\gamma \epsilon_{x}[\mu \mathrm{m}]$ & 30 & 0.66 & 10 & 100 & 50 & 0.04 \\
\hline$\gamma \epsilon_{y}[\mu \mathrm{m}]$ & 2 & 0.02 & 0.04 & 100 & 50 & 0.04 \\
\hline$e^{+}\left[10^{14} \mathrm{~s}^{-1}\right]$ & 0.06 & 1.1 & 3.9 & 18 & 440 & 100 \\
\hline
\end{tabular}




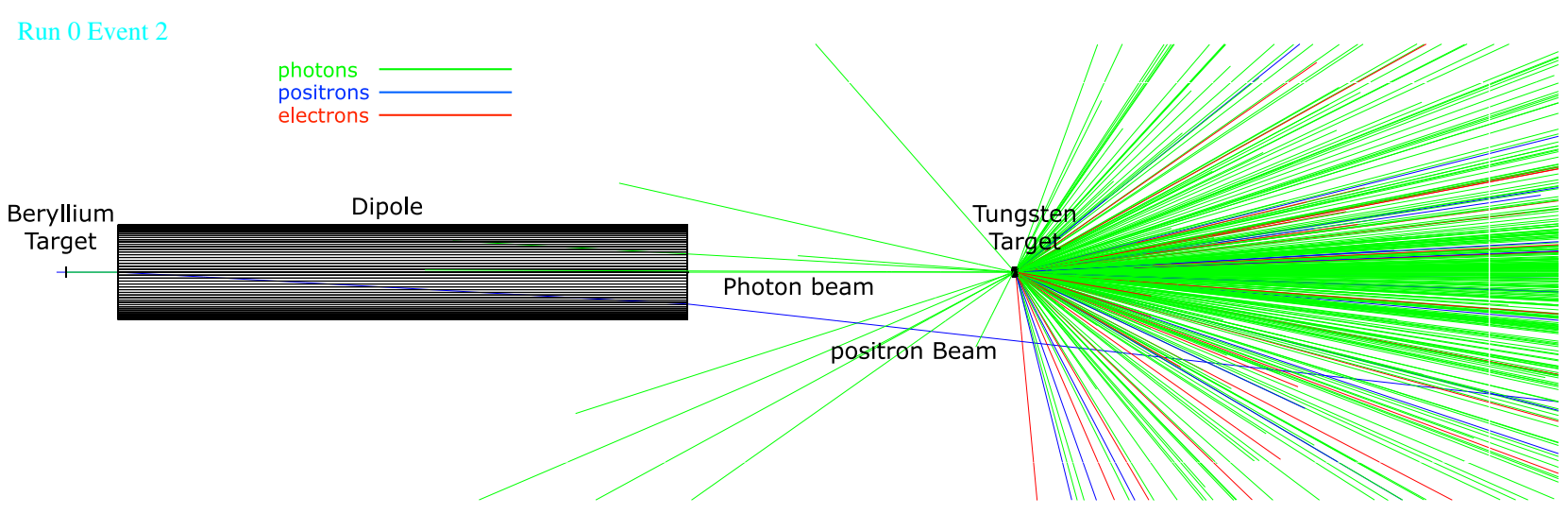

FIG. 13. Event display of GEANT4 for positron production.

embedded positron source. A thick high $\mathrm{Z}$ target placed downstream of the muon target can be used for electron positron pair production.

Experimental tests of such a scheme based on the adiabatic matching device collection scheme have been performed at KEK [20].

However we do not yet have a system that is able to transform the temporal structure of the produced positrons
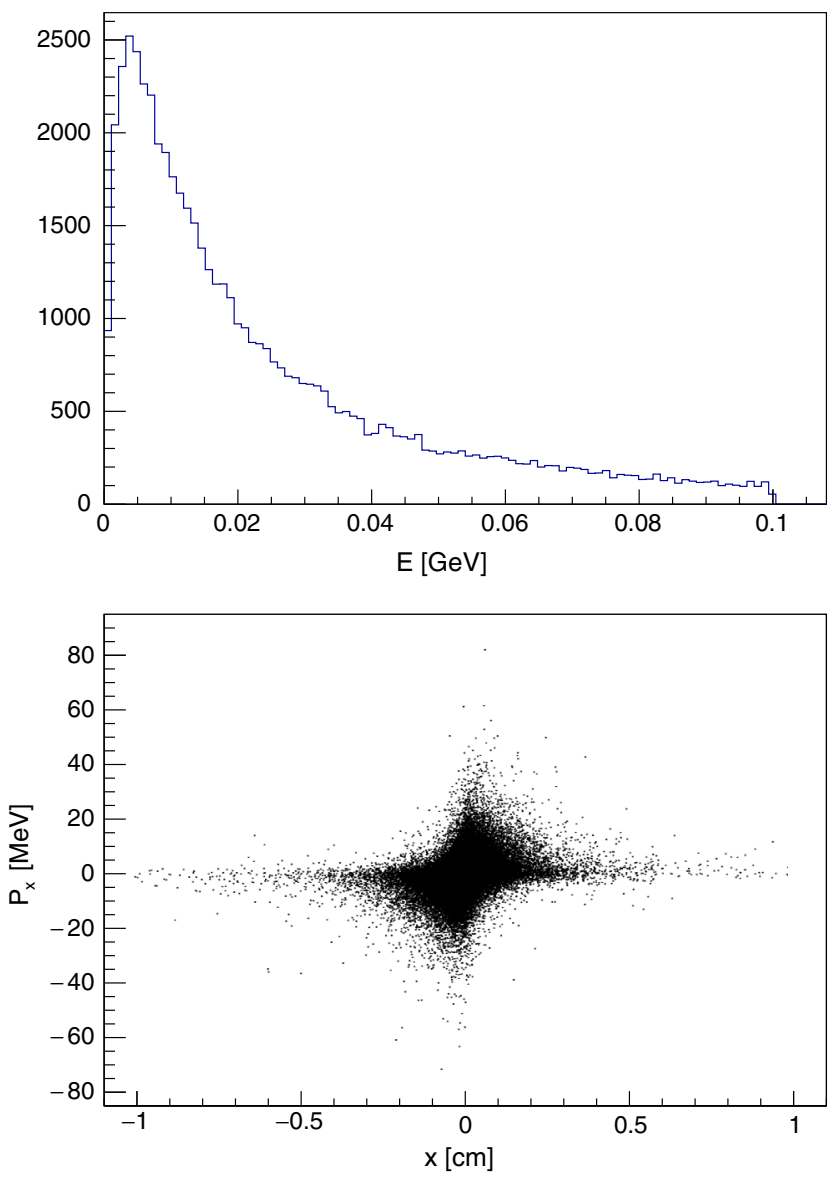

FIG. 14. Positrons energy spectra and transverse phase space distribution after the Tungsten target (GEANT4) from hitting photons produced at the muon target. to one that is compatible with the requirement of a standard positron injection chain. To evaluate the performance of such a scheme, a full Monte Carlo simulation has been performed, using GEANT4 for the part related to the positron production.

A simulation of the target system has been performed with GEANT4. The geometry set-up is shown in Fig. 13. A $45 \mathrm{GeV}$ monochromatic positron beam is sent towards a $3 \mathrm{~mm}$ Beryllium target. After the target, a dipole magnet of the positron ring bends away charged particles, while the produced photons proceed straight, interacting with the Tungsten target, where $\mathrm{e}^{+} \mathrm{e}^{-}$pairs are created.

It has been found that in the interaction of 100 positrons within the Beryllium target, about 11 photons and 5 electrons are created on average. As expected, photons are produced with a bremsstrahlung energy spectrum and strongly boosted along the beam axis. Only photons with high boost will reach the thick Tungsten target, with an average overall yield of about 10.5 photons per 100 primary positrons, with a very small $(<0.1$ per mill) contamination of residual $\mathrm{e}^{+} / \mathrm{e}^{-}$. In the interaction of these 10.5 photons in a $5 \mathrm{X}_{0}$ Tungsten target, about 65 positrons are produced, together with 77 electrons and 1200 photons. Figure 14 shows the positron energy spectra and the positron transverse phase space distribution.

In conclusion, the suggested scheme produces about 60 secondary positrons from the interaction of 100 primary positrons, of which less than $\sim 3$ are lost due to the interaction in the beryllium target. It would thus be enough to have a collection efficiency for these secondary positrons of about $5 \%$ in order to be able to recover the loss in the primary beam. A full simulation in GEANT4 and ASTRA [21] will be performed in future study, following the adiabatic matching scheme proposed in [22].

\section{CONCLUSION AND PERSPECTIVES}

We have presented a novel scheme for the production of muons starting from a positron beam on target, discussing some of the critical aspects and key parameters of this idea and giving a consistent set of possible parameters to show 
its feasibility. This scheme has several advantages, the most important one is that it can provide low emittance muons without adding cooling.

This innovative scheme has many key topics to be investigated: a low emittance $45 \mathrm{GeV}$ positron ring, $\mathrm{O}(100 \mathrm{~kW})$ class target, high rate positron source, and a $22 \mathrm{GeV}$ muon accumulator ring.

We presented the preliminary study of a $45 \mathrm{GeV}$ positron ring with a thin Beryllium target insertion. The ring has an high momentum acceptance allowing a lifetime of about 40 turns for a $3 \mathrm{~mm}$ Be target. Beam emittance growth due to the interaction with target has been observed. A dedicated cell has been designed to show that the emittance growth can be contained with proper optics parameters at the target location.

We have shown that this effect can be reduced by lowering the value of the $\beta$-function at the target location in addition to the minimization of the linear and high order dispersion terms. However stringent constraints on the beam size at the target are imposed by thermomechanical stresses, posing a lower limit on the emittance that can be obtained. Although an increase in emittance with respect to that shown in Fig. 2 seems unavoidable, it might be compensated with an increase of the positron beam energy up to $50 \mathrm{GeV}$ where a factor of two in the muon production rate can be obtained, at the cost of more than a factor four larger emittance with almost no loss in muon collider luminosity performance. Nevertheless, one has to cope with a much larger muon energy spread, up to $18 \%$.

Further improvements in nonlinear effects corrections to increase the energy acceptance of the positron ring can be studied. We need also to reduce the momentum compaction to be as close to zero as possible in order to decrease the positron-and thus also the muon-bunch length. Beam instabilities driven by this small momentum compaction need to be studied, as well.

The possibility of increasing the ring circumference has to be fully explored since it would allow the reduction of the synchrotron radiation power loss. Keeping the same number of positrons per bunch and the same bunch distance one would get the same muon production rate for the same positron source requirements. In addition, by increasing the ring circumference, the ring parameters could be improved, reducing transverse emittance and momentum compaction and increasing dynamical aperture and momentum acceptance. Progress need to include all other topics like target material, muon accumulation issues, positron source, and injection.

\section{ACKNOWLEDGMENTS}

The authors thank L. Keller for useful discussions and suggestions. The authors also thank I. Chaikovska and R. Chehab for discussions on the positron source and AMD scheme, S. Gilardoni and M. Calviani for suggestions about the target issues and $\mathrm{H}$. Burkhardt for discussions about GEANT4.
[1] The muon accelerator program, http://map.fnal.gov.

[2] M. Palmer, The US muon accelerator program, in Proceedings, 5th International Particle Accelerator Conference (IPAC 2014): Dresden, Germany, 2014 (2014), p. TUPME012, http://accelconf.web.cern.ch/accelconf/IPAC2014/papers/ tupme012.pdf.

[3] M. Antonelli, M. Boscolo, R. Di Nardo, and P. Raimondi, Novel proposal for a low emittance muon beam using positron beam on target, Nucl. Instrum. Methods Phys. Res., Sect. A 807, 101 (2016).

[4] M. Boscolo et al., Studies of a scheme for low emittance muon beam production from positrons on target, in Proceedings, 8th International Particle Accelerator Conference (IPAC 2017): Copenhagen, Denmark, 2017 (2017), p. WEOBA3, http://accelconf.web.cern.ch/ AccelConf/ipac2017/papers/weoba3.pdf.

[5] W. A. Barletta and A. M. Sessler, Characteristics of a highenergy $\mu^{+} \mu^{-}$collider based on electro-production of muons, Nucl. Instrum. Methods Phys. Res., Sect. A 350, 36 (1994); Characteristics of a high-energy $\mu^{+} \mu^{-}$ collider based on electro-production of muons, AIP Conf. Proc. 352, 31 (1996).

[6] B. Autin, A. Blondel, and J. R. Ellis, Prospective study of muon storage rings at CERN, Report No. CERN-1999-002.

[7] GEANT4, toolkit for the simulation of the passage of particles through matter, http://geant4.cern.ch.

[8] J.C. Biasci et al., A low-emittance lattice for the esrf, Synchrotron Radiat. News 27, 8 (2014).

[9] B. Nash, N. Carmignani, L. Farvacque, S. M. Liuzzo, T. Perron, P. Raimondi, R. Versteegen, and S. White, New functionality for beam dynamics in accelerator toolbox (at), in Proceedings, 6th International Particle Accelerator Conference (IPAC 2015): Richmond, VA, USA, 2015 (2015), http://accelconf.web.cern.ch/AccelConf/IPAC2015/ papers/mopwa014.pdf.

[10] MAD-X, Methodical Accelerator Design, http://mad.web .cern.ch/mad.

[11] FLUKA, fully integrated particle physics MonteCarlo simulation package, http://www.fluka.org/fluka.php.

[12] D. Neuffer, Principles and applications of muon cooling, Part. Accel. 14, 75 (1983).

[13] N. Munemoto et al., Direct injection of fully stripped carbon ions into a fast-cycling induction synchrotron and their capture by the barrier bucket, Phys. Rev. Accel. Beams 20, 080101 (2017).

[14] J.-G. Hwang, E.-S. Kim, H.-J. Kim, and D.-O. Jeon, Minimization of the emittance growth of multicharge particle beams in the charge stripping section of raon, Nucl. Instrum. Methods Phys. Res., Sect. A 767, 153 (2014).

[15] D Möhl, Sources of emittance growth, CERN Accelerator School and KVI: Specialised CAS Course on Small Accelerators, 2006 (Zeegse, The Netherlands, 2005), http://dx.doi.org/10.5170/CERN-2006-012.

[16] F. Maciariello et al., High intensity beam test of low Z materials for the upgrade of SPS-to-LHC transfer line collimators and LHC injection absorbers, in Proceedings, 7th International Particle Accelerator Conference (IPAC 2016): Busan, Korea, 2016 (Busan, Korea, 2016), p. TUPMB052, http://accelconf.web.cern.ch/AccelConf/ ipac2016/papers/tupmb052.pdf. 
[17] K. Ammigan et al., Experimental results of beryllium exposed to intense high energy proton beam pulses, in Proceedings, 2nd North American Particle Accelerator Conference (NAPAC2016): Chicago, Illinois, USA, 2016 (2017), p. MOPOB14, https://napac2016.aps.anl.gov/.

[18] E. Wakai et al., Engineering validation for lithium target facility of the ifmif under ifmif/eveda project, Nucl. Mat. Energy 9, 278 (2016).

[19] F. Zimmermann, O. S. Bruning, Y. Papaphilippou, D. Schulte, P. Sievers, V. Yakimenko, L. Rinolfi, A. Variola, F. Zomer, E. V. Bulyak, H. H. Braun, and M. Klein, Positron Options for the linac-ring LHEC, Proceedings of IPAC2012, New Orleans, Lousiana, USA (2012), WEPPR076, http://accelconf.web.cern.ch/accelconf/ipac 2012/papers/weppr076.pdf.

[20] I. Chaikovska et al., Optimization of an hybrid positron source using channeling, Nucl. Instrum. Methods Phys. Res., Sect. B 402, 58 (2017).

[21] K. Flottmann, ASTRA, A space charge tracking algorithm, http://www.desy.de/mpyflo/.

[22] R. Chehab, Angular collection using solenoids, Nucl. Instrum. Methods Phys. Res., Sect. A 451, 362 (2000). 